\title{
Thermodynamic analysis of energy density in pressure retarded osmosis: The impact of solution volumes and
}

\section{costs}

\author{
Kevin K. Reimund ${ }^{1}$, Jeffrey R. McCutcheon ${ }^{1}$, Aaron D. Wilson ${ }^{2} *$ \\ 1. University of Connecticut Department of Chemical and Biomolecular Engineering, Storrs, CT \\ 06269, USA \\ 2. Idaho National Laboratory, Idaho Falls, ID 83415-2208, USA
}

*corresponding author

Email: aaron.wilson@inl.gov

Phone: (208) 526-1103 


\begin{abstract}
A general method was developed for estimating the volumetric energy efficiency of pressure retarded osmosis via pressure-volume analysis of a membrane process. The resulting model requires only the osmotic pressure, $\pi$, and mass fraction, $w$, of water in the concentrated and dilute feed solutions to estimate the maximum achievable specific energy density, $u$, as a function of operating pressure. The model is independent of any membrane or module properties. This method utilizes equilibrium analysis to specify the volumetric mixing fraction of concentrated and dilute solution as a function of operating pressure, and provides results for the total volumetric energy density of similar order to more complex models for the mixing of seawater and riverwater. Within the framework of this analysis, the total volumetric energy density is maximized, for an idealized case, when the operating pressure is $\frac{\pi}{1+\sqrt{w}^{-1}}$, which is lower than the maximum power density operating pressure, $\frac{\Delta \pi}{2}$, derived elsewhere, and is a function of the solute osmotic pressure at a given mass fraction. It was also found that a minimum $1.45 \mathrm{kmol}$ of ideal solute is required to produce $1 \mathrm{kWh}$ of energy while a system operating at "maximum power density operating pressure" requires at least $2.9 \mathrm{kmol}$. Utilizing this methodology, it is possible to examine the effects of volumetric solution cost, operation of a module at various pressure, and operation of a constant pressure module with various feed.
\end{abstract}




\section{Introduction}

Pressure retarded osmosis is a method of generating energy from a potential energy gradient between two solutions [1-27]. In PRO, a volume of water is passed up a pressure gradient by a chemical potential gradient generated by dissolved solutes. The volume passed and its energy density are interdependent: operating at high pressures will limit the relative volume that can be passed but the volume will have a high energy density, while operating at low pressures will allow a larger relative volume of water to transfer with a lower energy density. There have been a number of papers which consider the energy that can be extracted in a PRO process; this energy is inversely related to the minimum energy of desalination [28-30]. Previous characterizations of volumetric energy density of PRO processes have defined a frame of reference to dictate the resulting value based on the stream perceived to be more expensive or limiting; two previous analyses of a commonly proposed osmotic energy source, the mixing of seawater and riverwater, resulted in different analyses by selecting opposite bases for normalization. Veerman et al investigate the "fuel efficiency" of a seawater-riverwater reverse electrodialysis (RED) process and concluded that if the pretreatment cost of riverwater is substantial [31], operating at high transfer by fully diluting the concentrated solution will be more efficient. Yip and Elimelech [14,15], however, arrive at an energy density of approximately $0.75 \mathrm{kWh} / \mathrm{m}^{3}$ of dilute feed solution for a seawater-riverwater PRO process by assuming the dilute feed solution will be the limiting resource. As the energy of mixing is a thermodynamic property, is has been shown that the maximum extractable energy for RED and PRO processes are identical for identical feed conditions [32]. More recently, Lin et al analyze the specific energy density of PRO process accounting for both feed and draw solution but ignoring, to an extent, dimensional parameters such as membrane area and module length [33]. By this type of 
analysis, the volumetric energy density of either or both streams may be specified to yield a more practical result for process design. A more general analysis of the "cost" per unit volume of PRO working solutions, such as pretreatment and pumping costs, allows extension of the concept of specific energy density to specific energy "cost", which allows the minimization of the total cost, per total volume of input solution.

Analyses of seawater and riverwater PRO processes have implied that the volumetric energy density of the process, which has a maximum value of approximately $0.192 \mathrm{kWh} / \mathrm{m}^{3}$ of seawater and riverwater combined, is too low, especially when considering pretreatment costs for seawater and riverwater, to be a practical source of renewable energy [33]. PRO has also been proposed and evaluated as an energy recovery process for reverse osmosis (RO), by which riverwater or treated wastewater is mixed with $\mathrm{RO}$ brine to recover a portion of the energy spent on desalination $[32,34,35]$. Both of these processes are open-loop, in that the feed streams are not manually regenerated. Osmotic heat engines (OHE) have been proposed which utilize continually regenerated (closed-loop) streams of concentrated and dilute solutions [33,36]. While such a system is feasible in a number of forms, OHE are typically contrived to utilize low-grade heat and are proposed as add-on energy recovery systems, not as primary energy generation technologies, as the thermal efficiencies are typically low and temperatures are restricted to those which polymeric membranes can withstand [37]. These engines are proposed as alternatives to organic Rankine cycle engines (ORC) for recovering waste heat from industrial processes and power production. A third form of PRO may be envisioned in which an external, natural source of feedwater, e.g. seawater or riverwater, is combined with a stored solution, e.g. rainwater or an specialized draw solute, to produce power. Such a system must only store one of the solutions of 
a traditional closed-loop PRO process. Like closed-loop systems, such "semi-open" systems must regenerate the initial stored solution and external solution for re-use and discharge.

The closed-loop and semi-open loop PRO processes allow for the storage of energy through the storage of the unmixed working fluids. A plant may operate as a load-leveling battery, generating concentrated and dilute solutions at night and mixing them during the day, or as a battery for peak demand or intermittent power production. For the application of an osmotic battery, the specific energy density allows the comparison between competing forms of stored energy which have better defined specific energy densities; this includes solid-state batteries, flow-cell battery working fluid, fuel cell fuels, and combustion fuels. The specific energy density, here defined only in terms of the process working fluids, may then be weighed in combination with other concerns, membrane power density, in $\mathrm{W} / \mathrm{m}^{2}$, the related module power density, in $\mathrm{W} / \mathrm{m}^{3}$, and the system cost in USD/W. By analyzing the specific energy density of osmotic agents as well as the limits on energy extraction by reasonable operating conditions, it is possible to compare PRO systems to competing technologies such as chemical heat pumps, thermal storage, pumped hydro gravity storage, solid-state batteries, flow cell batteries, and compressed air storage.

In this paper a general method is developed for estimating the volumetric energy efficiency of pressure retarded osmosis via pressure-volume analysis of a membrane process. The resulting model requires only the osmotic pressure, $\pi$, and mass fraction, $w$, of water in the concentrated and dilute feed solutions to estimate the maximum achievable specific energy density, $u$, as a function of operating pressure. This method utilizes equilibrium analysis to specify the volumetric mixing fraction of concentrated and dilute solution as a function of operating pressure, and provides results for the total volumetric energy density of similar order to 
more complex models. Utilizing this methodology, it is possible to examine the effects of volumetric solution cost, operation of a module at various pressure, and operation of a constant pressure module with various feed.

\section{Theory}

\subsection{Osmotic equilibrium and piston-type model of PRO processes}

The pressure required to maintain equilibrium between a solution of pure water and a solution containing a solute is the osmotic pressure. In a piston-type PRO process, as illustrated in Figure 1A, the initial state is that the applied pressure is equal to the osmotic pressure. If the pressure applied to the concentrated solution is changed by an infinitesimal amount, an infinitesimal amount of pure water will permeate the membrane. In all subsequent expressions, the water transferred $(\Delta V)$ is always treated relative to either the initial concentrated $\left(V_{H I}\right)$ or dilute $\left(V_{L I}\right)$ feed solutions. We assume proportionality between the osmotic pressure and molality (Morse equation), which is consistent over a wide range of concentrations relevant to PRO $[38,39]$ and modern draw solutes [40-51]. Inclusion of the initial mass fraction of water in the high osmotic pressure solution, $w_{H I}$, ensures that all transformations are conducted consistently with molality. The equilibrium state of a piston model, with a pure water feed, as a function of operating pressure is expressed by Equation 1.

$$
P_{e q}=\frac{\pi_{H I}}{\left(\frac{V_{H I}+\Delta V / w_{H I}}{V_{H I}}\right)}
$$

Integrating Equation 1 with respect to the volume transferred results in the product of pressure and volume, or the work $(W)$ done by the reversible piston PRO process. This equation 
simultaneously describes the ideal energy produced by a PRO process $(\Delta V>0)$ as well as the minimum energy required to desalination a volume of water to absolute purity $(\Delta V<0)$.

$$
W=\int_{0}^{\Delta V} \frac{\pi_{H I}}{\left(\frac{V_{H I}+\Delta V / w_{H I}}{V_{H I}}\right)} d \Delta V=V_{H I} \pi_{H I} w_{H I} \ln \left(1+\frac{\Delta V}{V_{H I} w_{H I}}\right)
$$

This alternative expression for the energy of mixing and method of calculating the minimum energy required for desalination is premised on the product of pressure and volume being an expression of energy. The volume-pressure model avoids the tabulated activity coefficients and a number of assumptions and approximations used in existing Gibbs free energy methods [28-30]. Equation 2 does not require the identity of the solute (or solutes) in solution and is perfectly capable of being applied to solution consisting of a collection of solutes such as seawater and most other solutions in need of treatment. The relationship is expected to be valid until at least a solutions eutectic or saturation concentrations due to the underlying osmotic relationships [38,39]. The simplicity of this method should allow for broad application in evaluating the fundamental energy cost of treating novel aqueous solutions or conducting a treatment outside the conventional $\sim 50 \mathrm{wt} \%$ dewatering of seawater.

In terms of the application of Equation 2 to PRO because the log term in Equation 2 is unbounded, the work done diverges to infinity for a PRO process given an infinite supply of pure water. Either practical operating condition of a) a fixed operating pressure or b) a non-zero feed osmotic pressure are sufficient to prevent the infinite evolution of energy. 

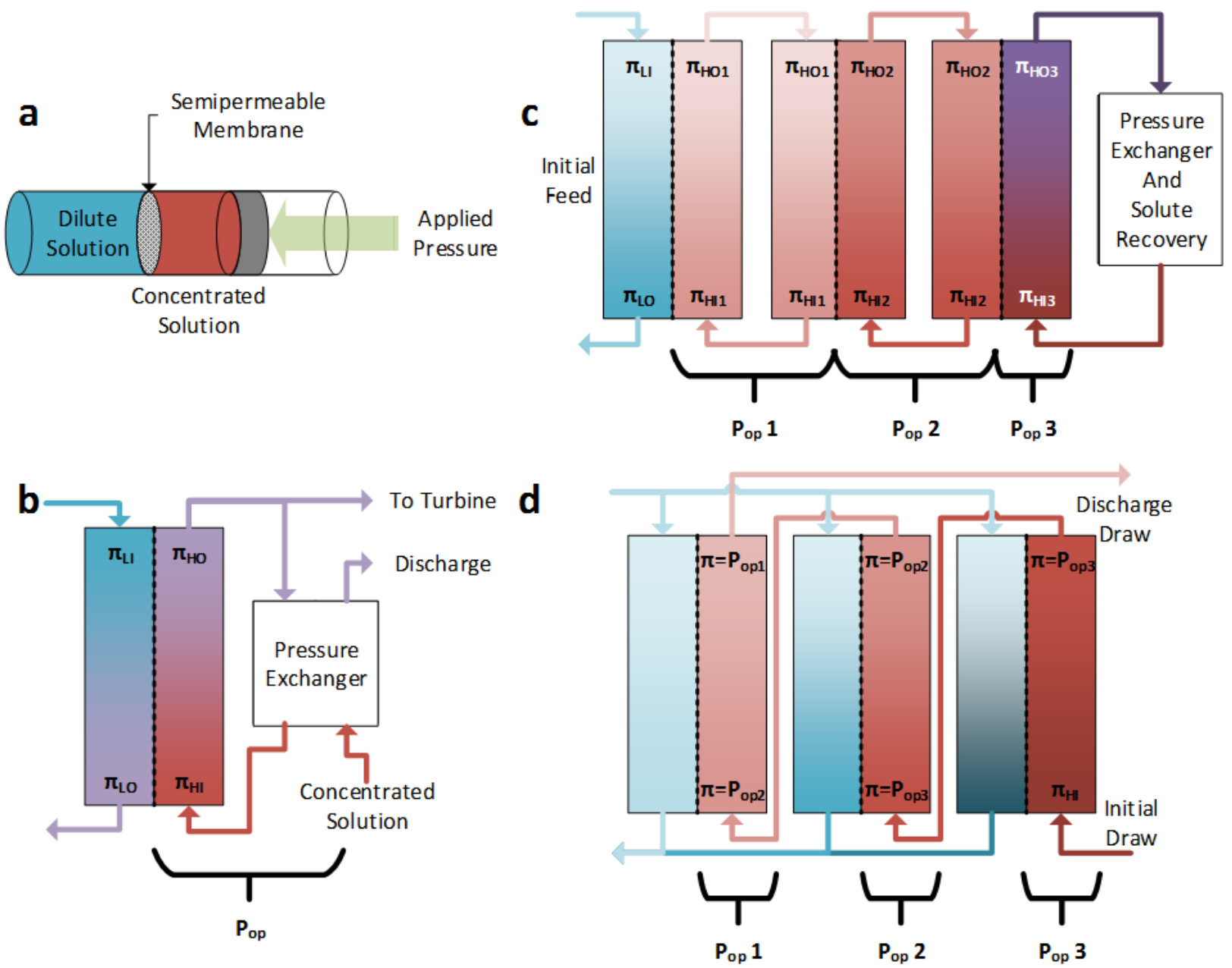

Figure 1: Four schemes of PRO operation. a) variable pressure ("piston-type") PRO. b) typical open-loop counter-current flow PRO process. c) A "series" PRO process with one dilute solution and staged concentrated solutions to access high osmotic pressures. d) A "parallel" PRO process with multiple dilute inlets and consecutive concentrated solution steps to approximate variable pressure operation, assuming each stage achieves equilibrium.

\subsection{Counter-flow PRO mass exchanger equilibrium}

It is impractical to operate variable pressure, i.e. piston-type, PRO systems for a number of reasons including a desire to achieve continuous operation in a practical implementation. 
Continuous PRO operation may be achieved in either co-current or counter-current flow, as in Figure 1B. The limiting case for co-current flow is equivalent mixing such that the output osmotic pressures are equal and features a decline in transmembrane osmotic pressure gradient along an element [33]. The corresponding limiting case for counter-current flow is that the output osmotic pressures are equivalent to the opposing input osmotic pressures with a more constant transmembrane osmotic pressure gradient is maintained along an element. The countercurrent flow can achieve the greatest degree of mixing possible for a constant-pressure system and can represent a perfect mass exchange system given infinite dimension [52]. A variablepressure PRO process may be approximated by operating subsequent modules as in Figure 1D at lower osmotic pressure, however it has been discussed [15] that the energy density per stage drops. Thus the power production per capital cost can be expected to decrease.

In the absence of both applied pressure and process non-idealities, the counter-current PRO process is reversible, despite operating within the framework of a fixed operating pressure. Real systems will not achieve osmotic equilibrium, since flux becomes low at low osmotic pressure differences and because real membranes are not perfectly selective; alternately, this is because, even for a perfect membrane, membrane area in a module is finite. A real PRO process will require an "excess" of the dilute solution or concentrated solution, depending on perspective, to account for process conditions and to drive process kinetics.

For a PRO process in which the feed osmotic pressure is not negligible, the operating pressure is no longer specified as in Equation 1, but rather as Equation 3.

$$
P_{e q}=\frac{\pi_{H I}}{\left(\frac{V_{H I}+\Delta V / w_{H I}}{V_{H I}}\right)}-\pi_{L I}
$$


and the work done is now expressed by Equation 4:

$$
W=\int_{0}^{\Delta V} \frac{\pi_{H I}}{\left(\frac{V_{H I}+\Delta V / w_{H I}}{V_{H I}}\right)}-\pi_{L I} d \Delta V=V_{H I} \pi_{H I} w_{H I} \ln \left(1+\frac{\Delta V}{V_{H I} w_{H I}}\right)-\pi_{L I} \Delta V
$$

The equilibrium outlet osmotic pressures can be found by rearranging Equation 3. In

Equations 5 and 6, the middle expressions define the equilibrium outlet osmotic pressures from the initial inlet state and the fixed operating pressure. The expressions on the right describe the equilibrium in terms of the equilibrium permeated volume $(\Delta V)$ and the initial states.

This analysis holds the trans-membrane pressure, the pressure directed to the pressure exchanger, and the pressure directed to the energy recovery turbine at an equivalent pressure represented by $P_{o p}$. This equivalence is made possible by fixing the volume directed to the pressure exchanger as $V_{H I}$ and the energy recovery turbine as $\Delta V$. During practical operation of a PRO system there may be reasons to let these volumes and pressures vary relative to each other but in this analysis it would introduce unresolvable variables without altering overall system properties and energies

$$
\begin{gathered}
\pi_{H O}=\left[\pi_{L I}+P_{O p}\right]=\frac{\pi_{H I}}{\frac{V_{H I}+\Delta V / w_{H I}}{V_{H I}}} \\
\pi_{L O}=\left[\pi_{H I}-P_{O p}\right]=\frac{\pi_{L I}}{\frac{V_{L I}+\Delta V / w_{L I}}{V_{L I}}}
\end{gathered}
$$

The equilibrium ratios of input volume to permeated volume may be calculated as Equations 7 and $\mathbf{8}$ where $\Delta \pi=\pi_{H I^{-}} \pi_{L I}$ is the difference in osmotic pressure between the input concentrated and dilute solution. 


$$
\begin{gathered}
\frac{V_{H I}}{\Delta V}=\frac{\pi_{L I}+P_{o p}}{w_{H I}\left(\Delta \pi-P_{o p}\right)} \\
\frac{V_{L I}}{\Delta V}=\frac{\pi_{H I}-P_{o p}}{w_{L I}\left(\Delta \pi-P_{o p}\right)}
\end{gathered}
$$

These ratios treat the operating pressure $\left(P_{o p}\right)$ as the primary independent variable while the volumes are dependent equilibrium or "minimal" values. This method side-steps many of the complications experienced when the dilution factor and operating pressure are varied in combination. Equations 7 and $\mathbf{8}$ can be combined to yield the ratio of the total volume to permeated volume as Equation 9.

$$
\frac{V_{H I}+V_{L I}}{\Delta V}=\frac{\pi_{H I} w_{H I}+\pi_{L I} w_{L I}+P_{o p}\left(w_{L I}-w_{H I}\right)}{w_{H I} w_{L I}\left(\Delta \pi-P_{o p}\right)}
$$

These "minimal" thermodynamic working volumes ratios can be converted to minimal inverse specific energy density, in $\mathrm{m}^{3} / \mathrm{kWh}$, by dividing by the transmembrane operating pressure; the term $P_{o p} \Delta V$ is the work done by the system. This converts Equations 7-9 to Equations 10-12.

$$
\begin{gathered}
\frac{V_{H I}}{P_{o p} \Delta V}=\frac{\pi_{L I}+P_{o p}}{P_{o p} w_{H I}\left(\Delta \pi-P_{o p}\right)} \\
\frac{V_{L I}}{P_{o p} \Delta V}=\frac{\pi_{H I}-P_{o p}}{P_{o p} w_{L I}\left(\Delta \pi-P_{o p}\right)} \\
\frac{V_{H I}+V_{L I}}{P_{o p} \Delta V}=\frac{\pi_{H I} w_{H I}+\pi_{L I} w_{L I}+P_{o p}\left(w_{L I}-w_{H I}\right)}{w_{H I} w_{L I} P_{o p}\left(\Delta \pi-P_{o p}\right)}
\end{gathered}
$$

If the dilute solution is assumed to be very dilute relative to the concentrated solution $\left(\pi_{L I} \ll \pi_{H I}\right)$, such that it can be assumed that $w_{L I} \approx 1, \pi_{L I} \approx 0$, and $\Delta \pi \approx \pi_{H I}$, then Equation 12 can be simplified and inverted to find the specific energy density as Equation 13. 


$$
\frac{P_{o p} \Delta V}{V_{H I}+V_{L I}} \approx \frac{w_{H I} P_{o p}\left(\pi_{H I}-P_{o p}\right)}{\pi_{H I} w_{H I}+P_{o p}\left(1-w_{H I}\right)}
$$

\section{Discussion}

\subsection{Advantage of osmotic pressure-volume method}

As mentioned the osmotic pressure-volume method assume an adaptation of the Morse relationship between osmotic pressure and concentrations [38,39]. Thus any future osmotic pressure resulting from the addition or removal of pure water can be predicted solely input osmotic pressure $\left(\pi_{H I}\right)$, the corresponding mass fraction $\left(w_{H I}\right)$, and relative volume of water added $V_{L I} / \Delta V$ and $V_{H I} / \Delta V$. The initial osmotic pressure is ideally derived from experimental measurement (i.e. vapor pressure, isopiestic, freezing point depression). This osmotic pressurevolume method is considerably simpler than expressions derived using the Gibbs free energy $[15,28,29,33,34]$, which explicitly make a variety of dilute-solution assumptions, limiting their extensibility. The results achieved using the osmotic pressure-volume method derived in this paper are comparable to dilute predictions derived from other models but can address far more concentrated solutions. As a result, the performance of a PRO system may be estimated from a limited number of initial states without reference to extensive tabulated data.

\subsection{Specific energy of draw solutes and feed solution}

An "ideal" solution containing a solute, when presented with a perfect membrane and infinite supply of pure water will dilute indefinitely. As demonstrated by Equation 2 the osmotic energy in this dilution is unbound and the recoverable energy is infinite for a variable pressure 
system. As has been demonstrated in other work [14,15], a fixed-pressure PRO process discards the energy of mixing above the operating pressure as well as the energy of the remaining unmixed solution below the operating pressure. The introduction of $P_{o p}$ not only reduces the osmotic energies to finite quantities but allows definition of the maximum specific energy densities $u_{\max }$.

Similar to the unbound state, the maximum energy density of the concentrated solution is realized when a large volume of dilute solution is used to allow complete mixing/dilution of the concentrated solution, such that $P_{o p}$ approaches its minimum. This state can be found by solving the inverse of Equation 10 for the case where the $P_{o p}$ approaches zero, Equation 14 (third expression). Assuming $\pi_{L I}$ also approaches zero and $P_{o p} \approx \pi_{L I}$ allows Equation 14 to be further reduced (fourth expression).

$$
u_{\max }^{H}\left(k W h / m^{3}\right)=\frac{P_{o p} \Delta V}{V_{H I}}=\frac{P_{o p} w_{H I}\left(\pi_{H I}-\pi_{L I}\right)}{\pi_{L I}} \approx \pi_{H I} w_{H I}
$$

The $u^{H}{ }_{\max }$ represent the specific energy contained in a unit volume of solution or solute that is diluted at a constant pressure and allows for comparison between different osmotic agents with knowledge of the initial osmotic pressure and water mass fraction. This value is commonly reported as the amount of energy in a draw solution [16] but would require an infinite volume of dilute solution to obtain.

All expressions of Equation 14 are proportionally dependent on the input volume of the concentrated solution, $V_{H I}$. The volume dependence of $u_{\max }^{H}$ is best described as $\mathrm{m}^{3}=V_{H I}$ while $\pi_{H I}$ can be substituted with $\mathrm{nRT} / V_{H I}$. Multiplying Equation 14 by the common denominator $V_{H I}$ and assuming at temperature of $298 \mathrm{~K}$ allows the proportional dependence to be removed to 
solve for exact number of moles of concentrated solute required per $\mathrm{kWh}$ as expressed by

Equation 15. This relationship indicates at least $1.45 \mathrm{kmol}$ of solute are required per $\mathrm{kWh}$.

$$
u_{\max }^{H}(k W h) \approx n R T w_{H I} \approx 0.688\left(k W h /_{k m o l}\right)\left(n \times w_{H I}\right)
$$

When energy density is described in terms of the low osmotic pressure solution, the maximum energy is released when $\Delta V \approx V_{L I}$ (i.e. complete mixing). Thus the operating pressure defines the energy density, $u^{L}$ Pop, as well as the energy requirements for $\mathrm{RO}$, of the dilute solution. This energy reaches a maximum when $P_{o p}=\Delta \pi \approx \pi_{H I}$ for a very dilute solution. This condition requires a unit volume of dilute solution to mix with an infinite volume of concentrated solution. The energy density of the dilute solution, as permeate, can be found by inverting Equation 11 and substituting $\Delta P_{o p}$ with $\pi_{H I}$ for Equation 16.

$$
u_{P_{o p}}^{L}\left(k W h /_{m^{3}}\right)=\frac{P_{o p} \Delta V}{V_{L I}} \approx P_{o p} w_{L I} \approx P_{o p} \frac{\Delta V}{\Delta V}
$$

When Equation 11 is used for a 0.6 M solution of sodium chloride at $298 \mathrm{~K}$, the osmotic pressure is approximately 26.3 bar and the corresponding $u^{L}{ }_{P o p}$ for a pure water feed is 0.73 $\mathrm{kWh} / \mathrm{m}^{3}$, which is comparable to other results [14]. This value is also the equivalent of the energy required for the removal of an infinitesimal volume of water from seawater.

\subsubsection{Percent energy recoveries for dilute and concentrated solutions}

The percentage of the maximum energy utilized by the input streams is the ratio of the energy at the specified operating condition to the maximum energy available for that stream.

For the concentrated solution this is the ratio of the inverse of Equation 10, to the $u^{H}{ }_{\max }$ for that solution, Equation 14, which yields the exact solution, Equation 17, and a simplified 
approximation when for dilute feeds when $\pi_{L I}$ approaches zero. Notably even for the exact solution, this relationship is independent of the weight fraction $w_{H I}$.

$$
u_{\text {recovered }}^{H I}(\%)=\frac{P_{o p}}{\pi_{H I}} \frac{\left(\Delta \pi-P_{o p}\right)}{\pi_{L I}+P_{o p}} \approx 1-\frac{P_{o p}}{\pi_{H I}}
$$

Similarly for the dilute solution, the fraction of available energy extracted is the ratio of the inverse of Equation 11 to Equation 13, when $P_{o p}=\pi_{H I}$, yielding the exact solution, Equation 18. For a dilute feed, $\pi_{L I}$ approaches zero, the relationship can be simplified.

$$
u_{\text {recovered }}^{L I}(\%)=\frac{P_{o p}}{\pi_{H I}} \frac{\left(\Delta \pi-P_{o p}\right)}{\pi_{H I}-P_{o p}} \approx \frac{P_{o p}}{\pi_{H I}}
$$

The total fraction of available energy extracted, or the efficiency, can be found by summing the energy extracted normalized to each stream divided by the volume fraction of that stream, i.e. $\frac{u_{\text {recovered }}^{H I} V_{H I}+u_{\text {recovered }}^{L I} V_{L I}}{V_{H I}+V_{L I}}$. Plotting the relative fraction of energy recovered relative to $\frac{P_{o p}}{\pi_{H I}}$ yields Figure 2 in which increases in the dilute solution osmotic pressure shift the maximum efficiency towards lower operating pressures. 




Figure 2: Percent energy utilization for the concentrated, dilute, and total (solid) system volumes for seawater simulant (26.3 bar osmotic pressure) and dilute stream osmotic pressure of 0,1 , and 2 bar (approximately 0, 20, and $40 \mathrm{mM} \mathrm{NaCl}$ at $298 \mathrm{~K}$ ). Please refer to online version to see the figure in color.

\subsection{Volume and Energy Optimization of PRO Processes}

Analyzing, by Equation 13, a solution of seawater simulant (26.3 bar, 96.5 wt\% water) and a dilute solution of 0.263 bar $(1 / 100)$ and $99.9 \mathrm{wt} \%$ water, the resulting maximum specific energy density is $174 \mathrm{Wh} / \mathrm{m}^{3}$ at $12.9 P_{o p}=12.9$ bar. If values are assumed to be $0.6 \mathrm{M}$ and 20 mM sodium chloride at $298 \mathrm{~K}-\sim 29.7$ and $\sim 0.99$ bar, respectively, via the Morse relations [38] - 
the resulting specific energy density is $182 \mathrm{Wh} / \mathrm{m}^{3}$ at $14.2 \mathrm{bar}$, which is comparable to the results from Lin et al [33] $\left(192 \mathrm{Wh} / \mathrm{m}^{3}\right)$. Thus a seawater-riverwater PRO plant would be expected to require approximately $2.7-2.9 \mathrm{~m}^{3}$ each of seawater and riverwater per $\mathrm{kWh}$ of energy, before accounting for process inefficiencies. Operating at a higher pressure to extract more energy for a given volume of riverwater input will greatly increase the volumetric requirements of seawater.

The volume requirements per $\mathrm{kWh}$ for concentrated solutions of $\mathrm{NaCl}$ at seawater conditions ( 26.3 bar), 62 bar, 103 bar, and 171 bar are shown in Figure 3A for dilution with pure water. In the case of a pure water feed, the ideal operating point can be found via differentiation of Equation 12 to be Equation 18.

$$
P_{o p, i d e a l}=\frac{\pi_{H I}}{1+\frac{1}{\sqrt{w_{H I}}}}
$$

This result indicates that the ideal operating pressure is a function of the concentrated solution's solute mass fraction required to achieve a given osmotic pressure. This value is lower than the maximum power density operating pressure, $\frac{\Delta \pi}{2}$, derived elsewhere. However $\frac{\Delta \pi}{2}$ serves a reasonable approximation for most applications. When $P_{\text {op,ideal }}$ conditions are applied to Equation 15 we find that at least $2.9 \mathrm{kmol}$ of high concentration solute are required per $\mathrm{kWh}$ of energy stored or generated. The cost lithium ion batteries for electric vehicle manufactures is speculated to be near $300 \mathrm{USD} / \mathrm{kWh}$ (early 2015). Based on this cost threshold (and assuming $P_{\text {op,ideal }}$ conditions) any osmotic energy storage system must use a solute that costs substantially less (likely an order of magnitude less) than a $100 \mathrm{USD} / \mathrm{kmol}$ of ideal solute to be able to compete with the current cost of large lithium batteries in the storage market. 
In Figure 3A, the mass fraction of water is linearly interpolated for data for sodium chloride at $298 \mathrm{~K}$ yielding $w_{w} \approx 1-0.001331 \pi$. Thus the osmotic pressures in Figure 3A approximately correspond to ideal operating pressures, of 13.03, $30 \mathrm{bar}, 50 \mathrm{bar}$, and $80 \mathrm{bar}$, respectively. While the ratio of volume required to minimum volume required are roughly identical for operation at a given operating pressure, the depression of volume per $\mathrm{kWh}$ at higher operating pressure allows for more flexibility in the operating condition. Subjectively, an additional $\mathrm{m}^{3}$ per $\mathrm{kWh}$, beyond the minimum $\mathrm{m}^{3} / \mathrm{kWh}$, might be a reasonable target for practical PRO operation; the region of operating pressures within this bound are shown in Figure 3A and decrease with decreasing osmotic pressure. This means that an osmotic power system using a higher osmotic draw has the flexibility to operate at higher pressure, with lower dilution of the draw solution, or lower pressure, with higher dilution of the draw solution.


Figure 3: A) Volume requirements per $\mathrm{kWh}$ for operation with various high-pressure draw solutions with pure water feed. Faint dashed lines indicate the operating condition at which one additional $\mathrm{m}^{3}$ per $\mathrm{kWh}$ is required. B) Constant-pressure total volume requirements per $\mathrm{kW}_{\mathrm{h}}$ for 
sodium chloride and pure water. The ideal operating condition (black line) is the operating pressure specified from Equation 19.

If, alternately, the maximum operating pressure of a module is known, then it is possible to choose the osmotic pressure of the concentrated solution for a closed loop system. Figure 3B shows the effect of changing the osmotic pressure of the concentrated solution, of sodium chloride, for fixed operating pressures of $13.04,30,50$, and 80 bar; these are representative feasible target operating pressures for PRO modules.

Similar to the variable pressure operating condition shown in Figure $\mathbf{3 A}$, the higher operating pressure makes the resulting system more insensitive to deviations from the ideal operating point generated via Equation 19, which represents variable pressure operation. Operating with osmotic pressures higher than 166 bar at a fixed operating pressure of 80 bar means the PRO process will operate to the left of the ideal operating point show in Figure 2, such that $P_{o p}<P_{o p \text {,ideal }}$. These conditions reduce the energy density of the dilute solution but increase the energy density of the concentrated solution. Because of the flattening that occurs at higher pressures, it is more feasible to operate an osmotic battery or osmotic heat engine at reduced pressures than it is to operate a seawater-riverwater PRO system. Figure 3B illustrates the effects of choosing the osmotic pressure for a module operating at a hypothetical maximum pressure for concentrated sodium chloride solutions. Higher operating pressures approximate better the ideal operating curve, and it appears from Figure $3 \mathbf{B}$ that $1 \mathrm{kWh} / \mathrm{m}^{3}$ is a feasible target energy density for a PRO process, as this is achievable with 50 bar operating pressure, which may be within the reach of existing membranes [37]. 
The operating pressure of a membrane module is subject to practical constraints on both transmembrane pressure and absolute operating pressure; for current RO modules, these are roughly equivalent, but it is likely that the transmembrane pressure is the more limiting factor for PRO [53]. The characteristics desirable in a PRO membrane (e.g. thin, non-tortuous, hydrophilic, high porosity [54]) are at odds with making membranes robust enough for use in PRO. Some investigations have found that $\mathrm{RO}$ membranes experience a decrease in permeability due to compaction [55], while studies on FO processes with pressurized feeds [53,56,57] or otherwise a comparison between membrane parameters derived from PDMP and ODMP experiments [58] have implicated pressure as having an effect on effective membrane salt and water permeability. Thin-film composite forward osmosis membranes tested at high pressure in PRO conditions have been observed to fail between 48.3 and 55.2 bar [37]. Even with further advances it is unreasonable to expect a full scale a single PRO stage can operate at any pressure significantly greater than the maximum employed in commercial RO today. This suggests a limit to the operating pressure of a PRO module of, optimistically, around 80 bar transmembrane pressurethe maximum stated operating pressure for the Dow SW30XHR-440i element, for example, is 1200 psig ( $~ 83$ bar) [59]. A PRO process operating at 80 bar with a pure water feed would require about $0.5 \mathrm{~m}^{3}$ of fresh water per kWh of energy. Operating near $P_{o p, i d e a l}$ would require $\sim 0.5 \mathrm{~m}^{3}$ of 171 bar concentrated solution for an energy to solution volumes volume ratio of 0.96 $\mathrm{kWh} / \mathrm{m}^{3}$. There is no theoretical reason to limit the concentrated solution to 171 bar. It is thermodynamically valid to use the most concentrated osmotic material available, such as an anhydrous salt. If, for example, the concentrated solution with an osmotic pressure of 375 bar is generated from anhydrous $\mathrm{NaCl}$ with all water derived from flux across the membrane the system would be operating at $78 \%$ of $u_{\max }^{H}$ at 80 bar based on Equation 15. Applying these 
conditions to Equation 17 indicates such a system would require $0.025 \mathrm{~m}^{3}$ of salt per $\mathrm{kWh}$ of energy which is small compared to the $0.5 \mathrm{~m}^{3}$ requirement of fresh water. This analysis indicates that $2.1 \mathrm{kWh} / \mathrm{m}^{3}$ represents an approximate maximum for which a single-module PRO process using might be bounded due to current transmembrane pressure tolerances of 80 bar.

\subsection{Cost optimization of PRO}

The "value", or volumetric cost, of an osmotic agent or feedwater stream is not something that can be known a priori. The cost per unit volume of taking a feed stream from a reservoir and either discharging or regenerating it is important as economic considerations could shift the ideal operating point for a PRO process. The volumetric cost of a stream, $c_{x}$, may consist of pumping duty, pretreatment costs, heat duty, mechanical losses, or any number of quantifiable parameters. The total value of the stream is specified by $c_{H I} V_{H I}+c_{L I} V_{L I}$.

Since most treatments of PRO mixing processes to date assume or suggest that the dilute stream will be more valuable, the total cost is normalized by the dilute solution volumetric cost to yield a single parameter, $C$, or $\frac{c_{H I}}{c_{L I}}$. This ratio can be applied to Equations $\mathbf{7}$ and $\mathbf{8}$ and summed to yield an expression similar to Equation 13, which specifies now the total cost, rather than total volume, per $\mathrm{kWh}$, normalized to the dilute solution cost.

$$
\frac{C V_{H I}+V_{L I}}{P_{o p} \Delta V}=\frac{\pi_{H I} w_{H I}+C \pi_{L I} w_{L I}+P_{o p}\left(C w_{L I}-w_{H I}\right)}{w_{H I} w_{L I} P_{o p}\left(\Delta \pi-P_{o p}\right)}
$$

When the ratio $C$ is near 1, the input streams are of nearly equal value; the result from Equation 16 is then similar to the result from Equation 20. If $C$ is less than one, then the dilute solution is of higher value than the concentrated solution, and a higher operating pressure is chosen than the 
operating pressure which maximizes the specific energy density. If $C$ is greater than 1 , the concentrated solution is of higher value, so the cost is minimized by operating at lower pressure to increase dilution, and thus utilization, of the concentrated solution.

Cost weighting shifts the operating pressure away from the maximum specific energy density point and therefore operating conditions which minimize cost may not maximize efficiency. Similar to Figure 3A, we can define a practical operating regime as the area which requires one additional $\mathrm{m}^{3}$ of input solution per $\mathrm{kWh}$ of energy produced. For the concentrated solutions in Figure 3A, the values of $C$ which optimize Equation $\mathbf{2 0}$ at the required pressure are solved numerically and shown in Table 1. As in Figure 3A, operation at high pressure allows wide variety in concentrated and dilute ratios for efficient operation. Table 1 demonstrates that an 80 bar PRO module would be able to accept concentrated solution cost ratios between 1:36.10 and 3610:1 given $1 \mathrm{~m}^{3} / \mathrm{kWh}$ excess. While this method does not specify whether operation at the cost-minimizing pressure will be economically feasible, it does indicate that high pressure modules should feasibly be able to operate at such a cost-minimizing point.

Table 1: Cost ratios necessary to operate at minimum cost within $1 \mathrm{~m}^{3} / \mathrm{kWh}$ of the minimum volume point in Figure 2.

\begin{tabular}{|c|c|c|c|}
\hline \multirow[t]{2}{*}{ Draw Solute } & \multirow{2}{*}{$\begin{array}{c}\text { Minimum } \\
\frac{\boldsymbol{V}_{\boldsymbol{H} \boldsymbol{I}}+\boldsymbol{V}_{\boldsymbol{L} \boldsymbol{I}}}{\boldsymbol{k} \boldsymbol{W h}} \\
{\left[m^{3} / k W h\right]}\end{array}$} & \multicolumn{2}{|c|}{$\begin{array}{c}\text { Cost Ratio at } \\
\frac{V_{H I, \text { min }}+V_{L I, \text { min }}+1\left(m^{3}\right)}{k W h}\end{array}$} \\
\hline & & $P_{c}>P_{i}$ & $P_{c}<P_{i}$ \\
\hline 23.6 bar (13.03 bar ideal) & 6.279 & $1: 5.19$ & 519:1 \\
\hline 62 bar (30 bar ideal) & 2.426 & $1: 11.23$ & $1123: 1$ \\
\hline 103 bar (50 bar ideal) & 1.507 & 1:19.65 & $1965: 1$ \\
\hline 171 bar ( 80 bar ideal) & 0.962 & $1: 36.10$ & 3610:1 \\
\hline
\end{tabular}




\subsection{Staged PRO Processes}

Many proposals for PRO processes have noted the incredible potential energies that can be generated with concentrated solutions. Accessing these high osmotic pressures are difficult due to the transmembrane pressure limitations previously discussed. There are a number of variations by which PRO process may be staged [60], and two are featured in Figure 1. In one, a volume of dilute solution may be passed "up" through a number of serial (relative to dilute solution) PRO stages (Fig 1c), each increasing in pressure and concentration, while the volume passing through each stage remains constant. This "upgrades" the energy density of the dilute solution and allows the PRO system to access high operating pressures by maintaining a constant transmembrane pressure. The other method is to pass the diluted high osmotic pressure solution into parallel (relative to dilute solution) PRO stages with lower $P_{o p}$ (Fig 1d) to increase the volumetric energy density of the concentrated solution.

\subsubsection{Analysis of "serial" staged performance}

The "serial" staged system does not recover pressure between stages, and because the operating pressure of each stage changes minimally while the flux is maintained constant, the energy produced in a "serial" staged system is roughly $n \cdot u_{L I, 1}^{\text {total }}$ relative to the dilute solution, where $n$ is the number of stages and $u_{L I, 1}^{\text {total }}$ is the specific energy density of the first stage. The "serial" staged system can operate with intermediate draw solutes, since only the top stage needs to be externally regenerated. In a real system, concentration polarization effects would make it difficult to achieve equilibrium.

The "serial" PRO staging scheme requires that the $\Delta V$ across all stages must be constant and the volume of inputs and outputs be matched, such that $V_{H O, n}=V_{L I, n+1}, V_{L O, n+1}=V_{H I, n}$. To 
maintain a constant $\Delta V$ and ensure that the streams are balanced, the membrane area and $P_{o p}$ can be modeled as equivalent for each stage. Table 2 presents a sample PRO scheme with modules operating at 50 bar and with various sodium chloride concentrations, reaching an ultimate $\pi_{H I, t o p}$ of 299.3 bar, starting from a $\pi_{L I, b o t t o m}$ of 0 bar. The operating pressure of a stage is the sum of the transmembrane pressures for all previous stages. A serial staged PRO process must be capable of pumping relatively large volumes of liquid at high pressure, requiring high-pressure high-flow pumps, and the modules used must be capable of withstanding high absolute pressures.

Table 2: Performance of a serial staged PRO process (Figure 1C). Transmembrane pressure and concentrated solution osmotic pressures are chosen to ensure consistency between the stages with a constant $\Delta V$.

\begin{tabular}{|c|c|c|c|c|c|c|}
\hline Stage & $\begin{array}{c}\text { Stage } \\
\text { Transmembrane } \\
\text { Pressure } \\
P_{o p, n}\end{array}$ & $\begin{array}{c}\text { Stage } \\
\pi_{L I}\end{array}$ & $\begin{array}{c}\text { Stage } \\
\pi_{H I}\end{array}$ & $\begin{array}{c}\text { Turbine } \\
\text { Operating } \\
\text { Pressure } \\
\sum P_{o p, n}\end{array}$ & $\frac{V_{L I, n}+V_{H I, n}}{V_{L I, 1}+V_{H I, 1}}$ & $\begin{array}{l}\frac{P_{o p} \Delta V}{V_{L I}} \\
\text { (Eq. 8) }\end{array}$ \\
\hline & [bar] & [bar] & [bar] & [bar] & {$\left[\mathrm{m}^{3} / \mathrm{m}^{3}\right]$} & {$\left[\mathrm{kWh} / \mathrm{m}^{3}\right]$} \\
\hline 1 & 50 & 0.00 & 103.9 & 50 & 1.0 & 1.389 \\
\hline 2 & 50 & 50.0 & 153.2 & 100 & $\sim 2.1$ & 2.778 \\
\hline 3 & 50 & 100 & 202.3 & 150 & $\sim 3.5$ & 4.167 \\
\hline 4 & 50 & 150 & 251.0 & 200 & $\sim 5.2$ & 5.556 \\
\hline 5 & 50 & 200 & 299.3 & 250 & 27.4 & 6.944 \\
\hline
\end{tabular}




\subsubsection{Analysis of "parallel" staged performance}

The "parallel" staged system recovers pressure, and thus energy, at each stage. Since each stage consumes "fresh" supply of dilute feed solution, while the "serial" staged system has only one input of dilute solution and one input of concentrated solution, the specific energy density relative to the total system volume will be much lower, but the specific energy density relative to the concentrated solution volume will be higher. Additionally, such a system would require a hydroturbine for each stage capable of recovering energy at each stage's pressure; this would considerably increase the capital cost of any plant.

Parallel staging instead requires that each stage be capable of generating power and repressurizing the concentrated feed to the previous one. Therefore each stage requires its own hydroturbine and pressure exchanger system. For a parallel system operating with an initial $\pi_{H I}$ of $171 \mathrm{bar}, \pi_{L I}$ of $1 \mathrm{bar}$, and a $P_{o p}$ of $80 \mathrm{bar}$, the results of a staging system are shown in Table

3.

Table 3: Performance analysis of a parallel staged PRO process (Fig 1d) with initial $\pi_{H I}$ of 171 bar and $\pi_{L I}$ of 1 bar.

\begin{tabular}{|c|c|c|c|c|c|c|c|}
\hline Stage & $\begin{array}{l}\text { Stage } \\
P_{o p, n}\end{array}$ & $\begin{array}{c}\text { Stage } \\
\pi_{H I}\end{array}$ & $\frac{V_{L I, n}}{V_{L I, 1}}$ & $\frac{\sum V_{L I, n}}{V_{L I, 1}}$ & $\frac{\sum \boldsymbol{V}_{L I, n}+\boldsymbol{V}_{H I}}{\sum \boldsymbol{P}_{o p, n} \Delta V_{n}}$ & $\frac{V_{H I}}{\sum V_{L I, n}}$ & $\frac{V_{H I}}{\sum P_{o p, n} \Delta V}$ \\
\hline & [bar] & [bar] & {$\left[\mathrm{m}^{3} / \mathrm{m}^{3}\right]$} & {$\left[\mathrm{m}^{3} / \mathrm{m}^{3}\right]$} & {$\left[\mathrm{m}^{3} / \mathrm{kWh}\right]$} & {$\left[\mathrm{m}^{3} / \mathrm{m}^{3}\right]$} & {$\left[m^{3} / k W h\right]$} \\
\hline 1 & 80.0 & 171 & 1.00 & 1.00 & 1.91 & 1.15 & 1.02 \\
\hline 2 & 38.9 & 81.0 & 1.93 & 2.93 & 1.89 & 0.38 & 0.52 \\
\hline 3 & 19.2 & 39.9 & 3.76 & 6.69 & 2.53 & 0.16 & 0.36 \\
\hline 4 & 9.55 & 20.2 & 7.28 & 13.98 & 3.83 & 0.078 & 0.28 \\
\hline 5 & 4.76 & 10.5 & 13.32 & 27.80 & 5.32 & 0.039 & 0.20 \\
\hline
\end{tabular}


The parallel staged system yields high energy densities when normalized to the draw solution. In stage 5 of Table $3,1 \mathrm{~m}^{3}$ of concentrated solution 171 bar yield a total of $5.02 \mathrm{kWh}$. However, the relative size of the total system volume for the staged system here is over 28 times greater than for the first stage alone. Therefore unless the cost ratio for the concentrated solution is very high, reduced pressure staging is likely to be economically unviable.

\subsection{Analysis of the osmotic battery}

The analysis presented here, as well as others, cast doubt on the feasibility of extracting energy from seawater-riverwater mixing through PRO due to the low specific energy density and expected pumping and pretreatment costs incurred in seawater-riverwater PRO. The specific energy density of seawater-riverwater PRO is low enough that pumping costs (e.g. far offshore pumping) or pretreatment can render the process unviable. An osmotic battery or osmotic heat engine can be designed for energy density or energy efficiency, making such a process feasible where seawater-riverwater PRO is not.

The demands of an osmotic heat engine are either good thermal efficiency or the ability to utilize very low quality heat sources. An osmotic heat engine might be employed as a primary power generator, powered via geothermal or solar energy, or as an add-on unit at existing chemical and power plants to utilize low-grade waste heat. An osmotic battery, conversely, is less dependent on overall efficiency than on the energy density of the system, its safety and stability, and the startup latency. In such a case, a highly energy dense solution or solute may be stored to maximize energy density and be regenerated, with less thermal efficiency, by some form of distillation or evaporation. A regeneration utilizing low-grade energy is useful, but not 
an absolute requirement for an osmotic battery. When electricity is abundant and less expensive it could be feasible to operate a RO-PRO process.

At the hypothetical operating limit presented in this work of 80 bar, an osmotic battery will have an energy density of $\sim 1 \mathrm{kWh} / \mathrm{m}^{3}\left(3.6 \mathrm{MJ} / \mathrm{m}^{3}\right)$. While this is less than typical for competitive systems like compressed air energy storage, pumped hydro, or flow batteries, an osmotic battery can be charged with low grade heat rather than electricity and is extremely scalable. The energy volumes can be increased by adding working fluid volumes and the power rates can be increased by adding additional PRO membrane elements and turbines.

\section{Conclusion}

This paper presents a methodology for evaluating the energy density of solution used in operating a PRO process as a function of the available solution osmotic pressures and the module operating pressure. Via this method, an alternative derivation the energy of mixing is reached in the form of Equation 2 and 4. Application of Equation 2 and 4 allows the calculation of thermodynamic limits for both osmotic energy generation processes and desalination processes without many of the approximations, limitations, and complications intrinsic in other models such as free energy methodologies. The extractable energy of counter-current PRO processes can be found through the same methodologies that provide the alternative derivation of energy is mixing. These methods also indicate the mole kmol quantity of solute required to produce a $\mathrm{kWh}$ unit of energy in an osmotic energy generation process depending on process effeincy. By defining the relative cost of the concentrated solution to the dilute solution, the ideal operating point can be redefined to allow optimization to minimize cost, rather than total solution volume.

The most commonly investigated form of PRO, the seawater-riverwater mixing process, contains relatively little energy per unit volume. $0.18-0.19 \mathrm{kWh} / \mathrm{m}^{3}$ are the maximum energy 
theoretically available for reasonable assumptions of salinity gradient (26.3-29.7 bar seawater, 0.263-1 bar dilute feed). When considering process inefficiencies including incomplete mixing, pressure exchanger inefficiency, and pumping losses. High-pressure PRO, through either osmotic batteries or osmotic heat engines, are not only capable of higher energy density, approaching and exceeding $1 \mathrm{kWh} / \mathrm{m}^{3}$, but are also free to operate within a wider range of conditions without significantly changing the absolute volume requirement as compared to lower pressure operation.

\section{Glossary of Terms:}

\begin{tabular}{|c|c|}
\hline \multicolumn{2}{|l|}{ Acronyms } \\
\hline FO & Forward osmosis \\
\hline $\mathrm{RO}$ & Reverse osmosis \\
\hline PRO & Pressure-retarded osmosis \\
\hline OHE & Osmotic heat engine \\
\hline ODMP & Osmotically driven membrane process \\
\hline PDMP & Pressure driven membrane process \\
\hline \multicolumn{2}{|l|}{ Symbols } \\
\hline$P$ & Pressure \\
\hline$P_{o p}$ & $\begin{array}{l}\text { transmembrane pressure (usually the turbine } \\
\text { pressure and pressure exchanger pressure) }\end{array}$ \\
\hline$\pi$ & Osmotic pressure \\
\hline$\Delta \pi$ & $\pi_{H I}-\pi_{L I}$ \\
\hline$w$ & Weight fraction of water \\
\hline$V$ & Volume \\
\hline$\Delta V$ & Volume of water transferred across membrane \\
\hline$c$ & Cost, per unit volume \\
\hline$C$ & Ratio of volumetric costs $\left({ }^{C} H I / c_{L I}\right)$ \\
\hline$S$ & Size, in total system volume, of a PRO stage \\
\hline \multicolumn{2}{|l|}{ Subscripts } \\
\hline$H I$ & High osmotic pressure stream input \\
\hline $\mathrm{HO}$ & High osmotic pressure stream output \\
\hline$L I$ & Low osmotic pressure stream input \\
\hline LO & Low osmotic pressure stream output \\
\hline$o p$ & Operating point \\
\hline i/ideal & $\begin{array}{l}\text { Ideal operating point (maximum specific energy } \\
\text { density) }\end{array}$ \\
\hline
\end{tabular}




\section{Acknowledgements}

This work was supported by the United States Department of Energy through contract DE-

AC07-05ID14517. Funding was supplied by Idaho National Laboratory via the Laboratory

Directed Research and Development program. The authors thank Prof. Nathan Tefft of Bates

College for discussion concerning the mathamatics.

\section{References}

[1] R.E. PATTLE, Production of Electric Power by mixing Fresh and Salt Water in the Hydroelectric Pile, Nature. 174 (1954) 660-660. doi:10.1038/174660a0.

[2] R.S. Norman, Water Salination: A Source of Energy, Science. 186 (1974) 350-352. doi:10.1126/science.186.4161.350.

[3] S. Loeb, R.S. Norman, Osmotic Power Plants, Science. 189 (1975) 654-655. doi:10.1126/science.189.4203.654.

[4] S. Loeb, Production of energy from concentrated brines by pressure-retarded osmosis : I. Preliminary technical and economic correlations, Journal of Membrane Science. 1 (1976) 49-63. doi:10.1016/S0376-7388(00)82257-7.

[5] H.H.G. Jellinek, H. Masuda, Osmo-power. Theory and performance of an osmo-power pilot plant, Ocean Engineering. 8 (1981) 103-128. doi:10.1016/0029-8018(81)90022-6.

[6] K.L. Lee, R.W. Baker, H.K. Lonsdale, Membranes for power generation by pressure-retarded osmosis, Journal of Membrane Science. 8 (1981) 141-171. doi:10.1016/S0376-7388(00)82088-8.

[7] S. Loeb, Energy production at the Dead Sea by pressure-retarded osmosis: challenge or chimera?, Desalination. 120 (1998) 247-262. doi:10.1016/S0011-9164(98)00222-7.

[8] J.W. Post, J. Veerman, H.V.M. Hamelers, G.J.W. Euverink, S.J. Metz, K. Nymeijer, et al., Salinitygradient power: Evaluation of pressure-retarded osmosis and reverse electrodialysis, Journal of Membrane Science. 288 (2007) 218-230. doi:10.1016/j.memsci.2006.11.018.

[9] S.E. Skilhagen, J.E. Dugstad, R.J. Aaberg, Osmotic power - power production based on the osmotic pressure difference between waters with varying salt gradients, Desalination. 220 (2008) 476-482. doi:10.1016/j.desal.2007.02.045.

[10] T. Thorsen, T. Holt, The potential for power production from salinity gradients by pressure retarded osmosis, Journal of Membrane Science. 335 (2009) 103-110. doi:10.1016/j.memsci.2009.03.003.

[11] Y. Xu, X. Peng, C.Y. Tang, Q.S. Fu, S. Nie, Effect of draw solution concentration and operating conditions on forward osmosis and pressure retarded osmosis performance in a spiral wound module, Journal of Membrane Science. 348 (2010) 298-309. doi:10.1016/j.memsci.2009.11.013.

[12] A. Achilli, A.E. Childress, Pressure retarded osmosis: From the vision of Sidney Loeb to the first prototype installation - Review, Desalination. 261 (2010) 205-211. doi:10.1016/j.desal.2010.06.017.

[13] G.Z. Ramon, B.J. Feinberg, E.M.V. Hoek, Membrane-based production of salinity-gradient power, Energy Environ. Sci. 4 (2011) 4423-4434. doi:10.1039/C1EE01913A. 
[14] N.Y. Yip, M. Elimelech, Performance Limiting Effects in Power Generation from Salinity Gradients by Pressure Retarded Osmosis, Environ. Sci. Technol. 45 (2011) 10273-10282. doi:10.1021/es203197e.

[15] N.Y. Yip, M. Elimelech, Thermodynamic and Energy Efficiency Analysis of Power Generation from Natural Salinity Gradients by Pressure Retarded Osmosis, Environ. Sci. Technol. 46 (2012) 52305239. doi:10.1021/es300060m.

[16] B.E. Logan, M. Elimelech, Membrane-based processes for sustainable power generation using water, Nature. 488 (2012) 313-319. doi:10.1038/nature11477.

[17] Y.C. Kim, M. Elimelech, Potential of osmotic power generation by pressure retarded osmosis using seawater as feed solution: Analysis and experiments, Journal of Membrane Science. 429 (2013) 330-337. doi:10.1016/j.memsci.2012.11.039.

[18] X. Wang, Z. Huang, L. Li, S. Huang, E.H. Yu, K. Scott, Energy Generation from Osmotic Pressure Difference Between the Low and High Salinity Water by Pressure Retarded Osmosis, Journal of Technology Innovations in Renewable Energy. 1 (2013) 122-130.

[19] I. Alsvik, M.-B. Hägg, Pressure Retarded Osmosis and Forward Osmosis Membranes: Materials and Methods, Polymers. 5 (2013) 303-327. doi:10.3390/polym5010303.

[20] C. Klaysom, T.Y. Cath, T. Depuydt, I.F.J. Vankelecom, Forward and pressure retarded osmosis: potential solutions for global challenges in energy and water supply, Chem. Soc. Rev. 42 (2013) 6959-6989. doi:10.1039/C3CS60051C.

[21] F. Dinger, Tr\&\#xf6, T. Ndle, U. Platt, Optimization of the Energy Output of Osmotic Power Plants, Journal of Renewable Energy. 2013 (2013) e496768. doi:10.1155/2013/496768.

[22] W. He, Y. Wang, M.H. Shaheed, Energy and thermodynamic analysis of power generation using a natural salinity gradient based pressure retarded osmosis process, Desalination. 350 (2014) 86-94. doi:10.1016/j.desal.2014.07.015.

[23] F. Helfer, C. Lemckert, Y.G. Anissimov, Osmotic power with Pressure Retarded Osmosis: Theory, performance and trends - A review, Journal of Membrane Science. 453 (2014) 337-358. doi:10.1016/j.memsci.2013.10.053.

[24] S.-S. Hong, W. Ryoo, M.-S. Chun, S.O. Lee, G.-Y. Chung, Numerical studies on the pressure-retarded osmosis (PRO) system with the spiral wound module for power generation, Desalination and Water Treatment. 52 (2014) 6333-6341. doi:10.1080/19443994.2013.821041.

[25] Z. Jia, B. Wang, S. Song, Y. Fan, Blue energy: Current technologies for sustainable power generation from water salinity gradient, Renewable and Sustainable Energy Reviews. 31 (2014) 91-100. doi:10.1016/j.rser.2013.11.049.

[26] A.P. Straub, S. Lin, M. Elimelech, Module-Scale Analysis of Pressure Retarded Osmosis: Performance Limitations and Implications for Full-Scale Operation, Environ. Sci. Technol. 48 (2014) 12435-12444. doi:10.1021/es503790k.

[27] M.G. Buonomenna, J. Bae, Membrane processes and renewable energies, Renewable and Sustainable Energy Reviews. 43 (2015) 1343-1398. doi:10.1016/j.rser.2014.11.091.

[28] K.S. Spiegler, Y.M. El-Sayed, The energetics of desalination processes, Desalination. 134 (2001) 109128. doi:10.1016/S0011-9164(01)00121-7.

[29] M. Elimelech, W.A. Phillip, The Future of Seawater Desalination: Energy, Technology, and the Environment, Science. 333 (2011) 712-717. doi:10.1126/science.1200488.

[30] L. Fitzsimons, B. Corcoran, P. Young, G. Foley, Exergy analysis of water purification and desalination: A study of exergy model approaches, Desalination. 359 (2015) 212-224. doi:10.1016/j.desal.2014.12.033.

[31] J. Veerman, M. Saakes, S.J. Metz, G.J. Harmsen, Reverse electrodialysis: Performance of a stack with 50 cells on the mixing of sea and river water, Journal of Membrane Science. 327 (2009) 136-144. doi:10.1016/j.memsci.2008.11.015. 
[32] B.J. Feinberg, G.Z. Ramon, E.M.V. Hoek, Thermodynamic Analysis of Osmotic Energy Recovery at a Reverse Osmosis Desalination Plant, Environ. Sci. Technol. 47 (2013) 2982-2989. doi:10.1021/es304224b.

[33] S. Lin, A.P. Straub, M. Elimelech, Thermodynamic limits of extractable energy by pressure retarded osmosis, Energy Environ. Sci. 7 (2014) 2706-2714. doi:10.1039/C4EE01020E.

[34] M.H. Sharqawy, S.M. Zubair, J.H. Lienhard V, Second law analysis of reverse osmosis desalination plants: An alternative design using pressure retarded osmosis, Energy. 36 (2011) 6617-6626. doi:10.1016/j.energy.2011.08.056.

[35] N.T. Hancock, N.D. Black, T.Y. Cath, A comparative life cycle assessment of hybrid osmotic dilution desalination and established seawater desalination and wastewater reclamation processes, Water Research. 46 (2012) 1145-1154. doi:10.1016/j.watres.2011.12.004.

[36] R.L. McGinnis, J.R. McCutcheon, M. Elimelech, A novel ammonia-carbon dioxide osmotic heat engine for power generation, Journal of Membrane Science. 305 (2007) 13-19. doi:10.1016/j.memsci.2007.08.027.

[37] A.P. Straub, N.Y. Yip, M. Elimelech, Raising the Bar: Increased Hydraulic Pressure Allows Unprecedented High Power Densities in Pressure-Retarded Osmosis, Environ. Sci. Technol. Lett. 1 (2014) 55-59. doi:10.1021/ez400117d.

[38] A.D. Wilson, F.F. Stewart, Deriving osmotic pressures of draw solutes used in osmotically driven membrane processes, Journal of Membrane Science. 431 (2013) 205-211. doi:10.1016/j.memsci.2012.12.042.

[39] A.A. Zavitsas, Properties of Water Solutions of Electrolytes and Nonelectrolytes, J. Phys. Chem. B. 105 (2001) 7805-7817. doi:10.1021/jp011053l.

[40] M.L. Stone, C. Rae, F.F. Stewart, A.D. Wilson, Switchable polarity solvents as draw solutes for forward osmosis, Desalination. 312 (2013) 124-129. doi:10.1016/j.desal.2012.07.034.

[41] A.D. Wilson, F.F. Stewart, Structure-function study of tertiary amines as switchable polarity solvents, RSC Adv. 4 (2014) 11039-11049. doi:10.1039/C3RA47724J.

[42] A.D. Wilson, C.J. Orme, Concentration dependent speciation and mass transport properties of switchable polarity solvents, RSC Adv. 5 (2015) 7740-7751. doi:10.1039/C4RA08558B.

[43] M.L. Stone, A.D. Wilson, M.K. Harrup, F.F. Stewart, An initial study of hexavalent phosphazene salts as draw solutes in forward osmosis, Desalination. 312 (2013) 130-136. doi:10.1016/j.desal.2012.09.030.

[44] C. Boo, Y.F. Khalil, M. Elimelech, Performance evaluation of trimethylamine-carbon dioxide thermolytic draw solution for engineered osmosis, Journal of Membrane Science. 473 (2015) 302309. doi:10.1016/j.memsci.2014.09.026.

[45] D. Li, H. Wang, Smart draw agents for emerging forward osmosis application, J. Mater. Chem. A. 1 (2013) 14049-14060. doi:10.1039/C3TA12559A.

[46] R. Ou, Y. Wang, H. Wang, T. Xu, Thermo-sensitive polyelectrolytes as draw solutions in forward osmosis process, Desalination. 318 (2013) 48-55. doi:10.1016/j.desal.2013.03.022.

[47] Q. Zhao, N. Chen, D. Zhao, X. Lu, Thermoresponsive Magnetic Nanoparticles for Seawater Desalination, ACS Appl. Mater. Interfaces. 5 (2013) 11453-11461. doi:10.1021/am403719s.

[48] J. Kim, J.-S. Chung, H. Kang, Y. Yu, W. Choi, H. Kim, et al., Thermo-responsive copolymers with ionic group as novel draw solutes for forward osmosis processes, Macromol. Res. 22 (2014) 963-970. doi:10.1007/s13233-014-2142-6.

[49] D. Zhao, S. Chen, P. Wang, Q. Zhao, X. Lu, A Dendrimer-Based Forward Osmosis Draw Solute for Seawater Desalination, Ind. Eng. Chem. Res. 53 (2014) 16170-16175. doi:10.1021/ie5031997.

[50] Y. Cai, W. Shen, R. Wang, W.B. Krantz, A.G. Fane, X. Hu, CO2 switchable dual responsive polymers as draw solutes for forward osmosis desalination, Chem. Commun. 49 (2013) 8377-8379. doi:10.1039/C3CC43289K. 
[51] Y. Hartanto, S. Yun, B. Jin, S. Dai, Functionalized thermo-responsive microgels for high performance forward osmosis desalination, Water Research. 70 (2015) 385-393. doi:10.1016/j.watres.2014.12.023.

[52] L.D. Banchik, M.H. Sharqawy, J.H. Lienhard V, Limits of power production due to finite membrane area in pressure retarded osmosis, Journal of Membrane Science. 468 (2014) 81-89. doi:10.1016/j.memsci.2014.05.021.

[53] B.D. Coday, D.M. Heil, P. Xu, T.Y. Cath, Effects of Transmembrane Hydraulic Pressure on Performance of Forward Osmosis Membranes, Environ. Sci. Technol. 47 (2013) 2386-2393. doi:10.1021/es304519p.

[54] J.R. Mccutcheon, M. Elimelech, Modeling water flux in forward osmosis: Implications for improved membrane design, AIChE J. 53 (2007) 1736-1744. doi:10.1002/aic.11197.

[55] M.T.M. Pendergast, J.M. Nygaard, A.K. Ghosh, E.M.V. Hoek, Using nanocomposite materials technology to understand and control reverse osmosis membrane compaction, Desalination. 261 (2010) 255-263. doi:10.1016/j.desal.2010.06.008.

[56] T. Yun, J.-W. Koo, J. Sohn, S. Lee, Pressure assisted forward osmosis for shale gas wastewater treatment, Desalination and Water Treatment. 0 (2014) 1-9. doi:10.1080/19443994.2014.941306.

[57] J. Duan, E. Litwiller, I. Pinnau, Solution-diffusion with defects model for pressure-assisted forward osmosis, Journal of Membrane Science. 470 (2014) 323-333. doi:10.1016/j.memsci.2014.07.018.

[58] A. Tiraferri, N.Y. Yip, A.P. Straub, S. Romero-Vargas Castrillon, M. Elimelech, A method for the simultaneous determination of transport and structural parameters of forward osmosis membranes, Journal of Membrane Science. 444 (2013) 523-538. doi:10.1016/j.memsci.2013.05.023.

[59] FILMTEC ${ }^{T M}$ SW30XHR-440i, (n.d.). http://www.dowwaterandprocess.com/en/products/f/filmtec_sw30xhr_440i (accessed January 13, 2015).

[60] W. He, Y. Wang, M.H. Shaheed, Enhanced energy generation and membrane performance by twostage pressure retarded osmosis (PRO), Desalination. 359 (2015) 186-199. doi:10.1016/j.desal.2014.12.014. 